erscheint in: van Riel R, Di Nucci E \& Schildmann J (Hrsg.). Enhancement der Moral. Mentis.

Ezio Di Nucci

\title{
Besser ist besser? Enhancement der Moral aus einer handlungstheoretischen Perspektive
}

Enhancement ist eine tolle Sache: dieser Begriff ist notwendigerweise positiv (ein bisschen wie der traditionelle Gottbegriff), so dass wenn eine Änderung keine richtige Verbesserung hervorbringt, es auch kein richtiges Enhancement gewesen ist: sehr praktisch. Wie könnte man unter diesen Umständen überhaupt gegen Enhancement sein? Beim Enhancement geht es nicht mal um das plausible aber nicht unumstrittene „mehr ist besser“; vielmehr geht es um das tautologische „besser ist besser“.

Die tautologische Natur von Enhancement ist besonders deutlich im Fall von moralischem Enhancement. Während man bei kognitivem Enhancement oder Neuroenhancement durchaus eine Kritik an dem "mehr ist besser" Prinzip versuchen könnte, wäre die gleiche Kritik begrifflich leer mit Bezug auf moralisches Enhancement; genauso leer wie die Negation des „besser ist besser" Prinzip. 
Eine ethische Analyse von moralischem Enhancement ist dann wenig interessant. Vielmehr geht es um die Frage, was überhaupt als moralisches Enhancement zählt, und das ist keine normative Frage im engeren Sinne. In diesem Kapitel möchte ich einen besonderen Aspekt dieser Frage untersuchen: welches Verständnis von moralischem Handeln wird vorausgesetzt, wenn wir annehmen, dass kognitives Enhancement oder Neuroenhancement auch in einer Art moralischem Enhancement resultieren kann? Was sagen wir über den Menschen als handelndem Wesen, wenn wir postulieren, dass eine Pille ihr Verhalten moralisch verbessern kann?

Folgendes scheint nicht unplausibel: wenn wir die kognitiven Fähigkeiten von Menschen steigern (zum Beispiel Aufmerksamkeit oder Denkgeschwindigkeit), dann werden wir diese Menschen auch besser ausrüsten, um Probleme zu lösen. Und das, so könnte man plausiblerweise meinen, ist genauso der Fall bei moralischen Problemen. Diese Menschen wären dann aus dem einfachen Grund moralisch verbessert, weil sie besser ausgerüstet wären, um moralische Probleme zu lösen.

Traditionellerweise gibt es wenigstens zwei Arten von Einwänden gegen dieses Bild von moralischem Handeln. Erstens ist die Lösung von moralischen Problemen nicht einfach eine Antwort, die man errechnen oder herausfinden kann. Die Lösung eines moralischen Problems beinhaltet auch, dass man 
handelt. Deswegen sind moralische Probleme nicht mit nichtmoralischen Problemen gleichzusetzen: wenn man herausgefunden hat, wie die Hauptstadt von Nicaragua heißt oder in welchem Jahr Bismarck geboren wurde, ist man gewissermaßen fertig: man hat die Aufgabe gelöst und es kann durchaus sein, dass eine gesteigerte Aufmerksamkeit die Lösung solcher Aufgaben unterstützen würde.

Moralische Probleme sind aber leider nicht schon gelöst, wenn man herausgefunden hat, welche Mittel das Problem heilen würden. Man muss dieses Mittel auch einsetzen. Oft wissen wir wirklich nicht, was wir zu tun haben. Noch öfter ist aber das Problem nicht, dass wir nicht wissen, was wir zu tun haben; Das Problem ist vielmehr unsere Motivation. In diesem Sinne sind moralische Probleme - wenn man sie unbedingt Probleme nennen möchte praktische Probleme und nicht (nur) theoretische Probleme. Man muss, vor allem, handeln; und die Lösungen von moralischen Problemen sind nie etwas anderes als Handlungen.

Der zweite Einwand besagt, dass der tugendhafte Akteur kein Problemlöser ist. Der tugendhafte Akteur muss nicht recherchieren oder herausfinden, was die moralische Antwort ist oder wie die Lösung eines moralischen Problems aussieht. Wenn jemand noch nach der Lösung suchen muss, dann ist dieser Mensch noch nicht tugendhaft. Tugend wäre dann die Fähigkeit, die Lösungen von moralischen Problemen unmittelbar und spontan zu erkennen. 
Nach Aristoteles handelt dann der tugendhafte Mensch gewohnheitsmäßig (moralisch) richtig: das heißt, dass es nicht reicht, einfach (moralisch) richtig zu handeln; man muss auch gewohnheitsmäßig (moralisch) richtig handeln. ${ }^{1}$ Die aristotelische Perspektive und ihre Relevanz für moralisches Enhancement zu verstehen ist das Hauptziel dieses Kapitels.

Eine für unsere Untersuchung entscheidende Stelle in der aristotelischen Nikomachischen Ethik lautet wie folgt (Buch II, 1105a14-b3):

Denn wo es sich um technische Fertigkeiten handelt, da hat das zustande gekommene Werk seine Angemessenheit in sich, und es genügt also, daß es so zustande kommt, daß es diese Eigenschaft irgendwie an sich trägt. Dagegen wo es sich um sittliche Betätigung handelt, da wird das Vollbrachte nicht schon dann etwa im Sinne der Gerechtigkeit oder der Besonnenheit vollbracht, wenn es diese Eigenschaften irgendwie an sich trägt, sondern es gehört dazu auch dies, daß der Handelnde auf Grund einer gewissen Form seiner Innerlichkeit tätig werde, und zwar zunächst, daß er mit Wissen, sodann daß er mit Vorsatz und

1 Siehe, unter anderem, Aristoteles 1925, Anscombe 1957, Rorty 1980, Burnyeat 1980, Charles 1984, Pollard 2003, Pollard 2006, Snow 2006, Peters (im Erscheinen), Rees \& Webber (im Erscheinen). 
zwar aus sachlichem Grunde, endlich drittens, daß er auch auf Grund einer zuverlässigen und unerschütterlichen Gesinnung seine Handlung vollziehe... Für die sittlichen Betätigungsweisen dagegen bedeutet das bloße Wissen wenig oder nichts, während die beiden übrigen Bedingungen hier nicht ein geringes, sondern geradezu alles bedeuten, und diese gelangen in unsere Gewalt eben durch das häufige Vollbringen gerechter und besonnener Handlungen. Handlungen werden also als gerecht und besonnen bezeichnet, wenn sie so vollbracht werden, wie ein gerechter oder besonnener Mann sie vollbringen würde. Und gerecht und besonnen ist nicht schon, wer solche Handlungen vollbringt, sondern erst, wer sie so vollbringt, wie Männer von gerechtem und besonnenem Charakter sie vollbringen. Und so sagt man denn mit Recht, daß man gerecht wird durch Vollbringen gerechter und besonnen durch Vollbringen besonnener Handlungen.

Folgende einfache Unterscheidung ist dann hilfreich, um die aristotelische Perspektive zu verstehen: es gibt richtige Handlungen und es gibt tugendhafte Handlungen. Während alle tugendhaften Handlungen notwendigerweise richtige Handlungen sind, sind nicht alle richtigen Handlungen tugendhaft. Die Bedingungen für Tugendhaftigkeit sind anspruchsvoller als die Bedingungen für (moralische) Richtigkeit. 
Wenn wir diese Unterscheidung einmal getroffen haben, kann die aristotelische Perspektive auf Enhancement folgendermaßen vereinfacht werden: wir können vielleicht durch pharmakologisches Eingreifen die menschliche Fähigkeit steigern, richtig zu handeln. Eine andere Frage ist aber, ob wir durch pharmakologisches Eingreifen auch die menschliche Fähigkeit, tugendhaft zu handeln, steigern können. Letzteres wird schwieriger.

Die Grundidee ist ganz einfach: überlegen oder nachdenken zu müssen, was in einer bestimmten Situation das Richtige zu tun wäre, ist ein Zeichen, dass man noch kein moralischer Experte ist; Experten müssen nicht überlegen oder nachdenken, weil sie schon wissen, was zu tun ist. Dazu kommt, dass das Überlegen an sich Kosten trägt: Nachdenken nimmt Zeit in Anspruch; Nachdenken nimmt kognitive Ressourcen in Anspruch; und es gibt klarerweise auch ein Restrisiko, dass das Überlegen zu falschen Schlussfolgerungen führen könnte. Wir haben also viele Gründe, warum es besser wäre, nicht überlegen oder nachdenken zu müssen. ${ }^{2}$

Die obengenannten Gründe sprechen dafür, dass ein nachdenkender Akteur kein Experte ist: es sind aber keine moralischen Gründe gegen

2 Für eine ausführliche Diskussion, siehe: Di Nucci 2008, Di Nucci 2011a, Di Nucci 2011b, Di Nucci 2012, Di Nucci 2013a, Di Nucci 2013b, Di Nucci 2013c, Di Nucci 2014a, und Di Nucci 2014b. 
Tugendhaftigkeit im engeren Sinne. Aber nachzudenken ist auch eine Form des Zögerns. Wer sich über das Gute sicher ist, sollte nicht zögern. Es ist nicht nur, wie oben, so, dass Zögern gefährlich sein kann (wobei es natürlich auch Vorteile haben kann); es ist vielmehr so, dass Zögern zeigt, dass man nicht voll bei der Sache ist, entweder weil man sich nicht sicher ist oder weil man Angst hat, oder vielleicht, weil man von den Vorteilen, sich an Regeln oder Gesetze nicht zu halten, angetan ist.

Nachdenken als Zögern ist dann eine Charakterschwäche, so dass der nachdenkende Akteur durchaus richtig handeln kann, aber (noch) nicht tugendhaft ist. Es ist aber klarerweise kein Zufall, dass der Experte spontan und regelmäßig immer das Richtige tut: diese Leistung ist das Resultat von Übung und Wiederholung, von einer gelungenen Erziehung und, wenn man so will, von einer langen Tugendausbildung (Dreyfus 1988 \& 2005; McDowell 1994).

Daher ist die gewohnheitsmäßige Natur von tugendhaften Handlungen so wichtig ${ }^{3}$ : daran merken wir, dass solche Handlungen nun in der Natur des Akteurs liegen; dass der Akteur im Einklang mit seinem Charakter handelt und nicht, zum Beispiel, gegen seine Natur oder seinen Charakter handelt. Es ist, in

3 "I want to claim that all virtuous actions are habitual in the above sense. That is not to say that habituality is all there is to virtuous action. Virtuous actions may have other characteristics, such as their having a certain goal, or being constituents of a good life. But habituality is a necessary, though not sufficient, condition of virtuous action." (Pollard 2003: 416). 
diesem Sinne, auch eine Frage der Wahrhaftigkeit. ${ }^{4}$ Diese Perspektive steht einer anderen alten ethischen Tradition kritisch gegenüber - die oft einerseits mit dem Katholizismus, anderseits mit Kant identifiziert wird - wobei sich überwinden zu müssen, Opfer zu bringen, ein besonderer moralischer Wert zugeschrieben wird.

Wir haben bislang dafür argumentiert, dass moralisches Handeln spontan und gewohnheitsmäßig sein muss und dass nachzudenken durchaus ein Zeichen von Charakterschwäche sein kann. Die Frage ist nun, welche Konsequenzen für moralisches Enhancement diese Auffassung haben könnte.

Lassen Sie uns mit einem Beispiel anfangen: ich habe morgen einen wichtigen Termin und ich möchte sicher gehen, dass ich den Leuten, die ich dort treffe, respektvoll und aufmerksam gegenüber auftreten werden. Dafür gibt es, so nehmen wir an, eine Pille. Eine Pille, damit ich zu anderen Leute netter sein kann. Oder stellen Sie sich vor, es gäbe eine Pille, die uns großzügiger machen würde. Das wäre doch was!

4 "Aristotle's idea is that the virtuous person is the one who naturally opts for the good deed; the one who doesn't have to decide or deliberate over which is the good deed. The virtuous deed is, in short, the one that the agent does not need to think about: it is only when virtue becomes second nature that the agent becomes virtuous. The agent, in a slogan, can't choose virtue: she must be virtuous, as the result of having been habituated to virtue in her upbringing." (Di Nucci 2008: 12). 
Lassen wir momentan die Frage beiseite, wie plausibel eine solche Pille ist: es ist zum Beispiel nicht undenkbar, dass man pharmakologisch Menschen empathischer machen könnte und damit vielleicht respektvoller oder großzügiger. ${ }^{5}$ Die Frage ist vielmehr: würde eine solche Pille meine Handlungen moralischer - im Aristotelischen Sinne - machen? Man könnte sich sogar vorstellen, dass eine solche Pille langfristige Auswirkungen auf mein Verhalten haben könnte: ich bin nicht nur morgen respektvoller oder großzügiger, ich bin langfristig respektvoller oder großzügiger (vielleicht muss ich dafür die entsprechende Pille einfach nur regelmäßig zu mir nehmen). Diese Beispiele 5 Hier ist ein Beispiel einer wissenschaftlichen Studie, die in eine ähnliche Richtung geht: „we show that the neurotransmitter serotonin directly alters both moral judgment and behavior through increasing subjects' aversion to personally harming others. We enhanced serotonin in healthy volunteers with citalopram (a selective serotonin reuptake inhibitor) and contrasted its effects with both a pharmacological control treatment and a placebo on tests of moral judgment and behavior. We measured the drugs' effects on moral judgment in a set of moral 'dilemmas' pitting utilitarian outcomes (e.g., saving five lives) against highly aversive harmful actions (e.g., killing an innocent person). Enhancing serotonin made subjects more likely to judge harmful actions as forbidden" (Crockett et al. 2010). Für eine philosophische Auseinandersetzung mit dieser Studie, siehe Harris\&Chan 2010 und Chan\&Harris 2011: “...serotonin may in fact be a moral de-enhancer, inhibiting or by-passing moral judgement. In the experiments, citalopram seemed to reduce participants' ability to reason about moral dilemmas in order to reach normative conclusions about the right action or outcome. From the reported research, the effect of serotonin seems to be to make subjects more responsive to immediate emotional engagement and less likely to reason beyond their instant protective reaction... it impairs or short circuits moral reasoning and induces us to act on the basis of emotion rather than rational consideration of the moral and social consequences of those actions." (Chan\&Harris 2011: 130). 
sind auch deswegen ausgewählt worden, weil die Steigerung von kognitiven Fähigkeiten wie Aufmerksamkeit oder Denkgeschwindigkeit in diesem Falle keine Rolle spielt: es geht nicht um die Lösung von schwierigen moralischen Dilemmata.

Jetzt ist die Frage, ob eine solche Pille mein Verhalten wirklich moralisch verbessern würde. Lassen Sie uns einfach die Annahme schenken, dass mein geändertes Verhalten als großzügiger und respektvoller bezeichnet werden kann (zum Beispiel von Außenstehenden, die nichts über diese Pille wissen). Es wäre vermutlich fair, in diesem Fall kontrafaktisch, zu sagen, dass ich mich nicht großzügiger verhalten hätte, wenn ich die Pille nicht genommen hätte. Aber ist das ein Problem? Moralische Gründe sind fast immer solche, von denen man kontrafaktisch sagen kann, dass man ohne den Grund anders gehandelt hätte.

Problematischer wäre, wenn wir davon ausgehen müssten, dass die Pille eine Art Zwang wäre: aber auch für diese Annahme sehe ich keinen Grund. Die Tatsache, dass die Pille mein Verhalten beeinflusst heißt nicht, dass dieser Einfluss zwanghaft ist: die meisten Einflüsse sind in der Tat nicht zwanghaft.

Was spricht dann gegen die These, dass die Pille eine echte moralische Verbesserung hervorbringt? Was dagegen spricht ist zum Beispiel, dass wir sicher nicht sagen können, dass zu meinem Charakter nun plötzlich 
Großzügigkeit gehört. Und wir können auch nicht sagen, dass ich nun gewohnheitsmäßig großzügig handele oder handeln werde. Großzügigkeit liegt noch nicht in meiner Natur, nur weil ich heute Morgen eine Pille geschluckt habe.

Wir sehen nun, dass die aristotelische Unterscheidung zwischen richtigen Handlungen und tugendhaften Handlungen, die wir in diesem Kapitel getroffen haben, für die Bewertung von moralischem Enhancement sehr hilfreich wird. Wir können diese Unterscheidung anwenden, um zu sagen, dass durch die Pille eine echte moralische Steigerung hervorgebracht wird, so dass ich nun wegen der Pille mich richtig oder auf jedem Fall besser verhalte. Aber die Pille ist nicht genug, um sagen zu können, dass ich nun tugendhaft bin: ich bin nicht wirklich großzügiger geworden, auch wenn ich großzügiger gehandelt habe. Die Pille hat etwas bewirkt aber ich habe noch nicht die Tugend der Großzügigkeit erworben. 


\section{Literatur}

Anscombe GEM (1957). Intention. Basil Blackwell, Oxford

Aristoteles (1969). Nicomachische Ethik. Reclam, Stuttgart

Burnyeat MF (1980). Aristotle on learning tob e good. In: Rorty AO (Hrsg.). Essays on Aristotle's Ethics. University of California Press, S. 69-92

Chan S, Harris J. (2011). Moral enhancement and pro-social behaviour. Journal of Medical Ethics. 37:130-1

Charles D. 1984. Aristotle's Philosophy of Action. Cornell University Press

Crockett MJ, Clark L. Hauser MD et al. (2010). Serotonin selectively influences moral judgment and behavior through effects on harm aversion. Proceedings of the National Academy of Sciences 107:17433-8

Di Nucci E (2008). Mind Out of Action. VDM, Saarbrücken

Di Nucci E (2011a). Frankfurt versus Frankfurt: a new anti-causalist dawn. Philosophical Explorations. 14: 117-31

Di Nucci E (2011b). Automatic Actions: Challenging Causalism. Rationality Markets and Morals. 2:179-200

Di Nucci E (2012) Priming Effects and Free Will. International Journal of Philosophical Studies. 20:725-734

Di Nucci E (2013a.) Mindlessness. Cambridge Scholars, Newcastle, UK

Di Nucci E (2013b). Habits, Nudges, and Consent. American Journal of Bioethics. 13:27-29

Di Nucci E (2013c). Action, Deviance, and Guidance. Abstracta. 7: 41-59

Di Nucci E (2014a). Aristotle and Double Effect. Journal of Ancient Philosophy. 8: 20-48

Di Nucci E (2014b). Ethics Without Intention. Bloomsbury, London

Dreyfus H. (1988). The Socratic and Platonic Bases of Cognitivism. Al \& Society. 2:99-112 
Dreyfus H (2005). Overcoming the Myth of the Mental: How Philosophers Can Profit from the Phenomenology of Everyday Expertise, APA Pacific Division Presidential Address

Harris J, Chan S (2010). Moral behavior is not what it seems. Proceeding of the National Academy of Sciences USA 107:E183.

McDowell J (1994). Mind and World (with a new introduction by the author: 1996). Harvard University Press, Cambridge (Mass.)

Peters J. (im Erscheinen). On Automaticity as a Constituent of Virtue. Ethical Theory and Moral Practice.

Pollard B (2003). Can Virtuous Actions Be Both Habitual and Rational?', Ethical Theory and Moral Practice. 6:411-25

Pollard B (2006). Explaining Actions with Habits. American Philosophical Quarterly. 43:57-68

Rees CF, Webber J. (im Erscheinen). Automaticity in Virtuous Action. In: Snow $\mathrm{N}$, Trivigno $\mathrm{F}$ (Hrsg.). The Philosophy and Psychology of Character and Happiness. Routledge, London

Rorty AO (Hrsg.) (1980). Essays on Aristotle's Ethics. University of California Press

Snow N (2006). Habitual Virtuous Actions and Automaticity. Ethical Theory and Moral Practice. 9:545-561 